\title{
FUNDAMENTOS DEL NEUROMARKETING DESDE LA NEUROCIENCIA DEL CONSUMIDOR PARA LA GENERACIÓN DE CONFIANZA
}

\author{
Tinoco-Egas, Raquel ${ }^{1}$ \\ Universidad Técnica de Machala, Ecuador \\ rmtinoco@utmachala.edu.ec
}

Material original autorizado para su primera publicación en la revista académica REDMARKA. Revista Digital de Marketing Aplicado.

https://doi.org/10.17979/redma.2016.01.016.4870

Recibido: 19 Mayo 2016

Aceptado: 20 Junio 2016

Resumen

Este documento recopila, a través de una revisión bibliográfica, los fundamentos del neuromarketing, desde la neurociencia del consumidor, para la generación de confianza a través de los medios sociales digitales. Concluye con tres principios aplicables en Neuromarketing para la generación de confianza; sensaciones de compra inteligente, influencia de la recomendación y contenidos de empatía que incluso puedan generar acciones colectivas.

Palabras claves: confianza, neuromarketing, neurociencia del consumidor, medios sociales digitales.

\footnotetext{
$\overline{1}$ RMTINOCO@UTMACHALA.EDU.EC MASTER OF INTERNATIONAL BUSINESS DEVELOPMENT, M SC. DOCTORADO EN ANÁLISIS ECONÓMICO Y ESTRATEGIA EMPRESARIAL (C.) NEGOCIOS ELECTRÓNICOS Y PUBLICIDAD (ORORESEARCH). UNIDAD ACADÉMICA DE CIENCIAS EMPRESARIALES, UNIVERSIDAD TÉCNICA DE MACHALA, AV. 25 DE JUNIO KM. 5 11/2 VÍA PASAJE, 070222 MACHALA, ECUADOR.

RAQUEL.TINOCO@UDC.ES

MASTER OF INTERNATIONAL BUSINESS DEVELOPMENT, M SC. DOCTORADO EN ANÁLISIS ECONÓMICO Y ESTRATEGIA EMPRESARIAL (C.) Investigación en Marketing Aplicado (iMARKA). Departamento en análisis económico y administración de empresas. Facultad de Economía y Empresa. Universidade da Coruña, Campus de Elviña, A Coruña 15071, España.
} 


\section{FUNDAMENTALS OF NEUROMARKETING FROM CONSUMER NEUROSCIENCE TO GENERATE TRUST}

\section{Abstract}

Tinoco-Egas, Raquel

This document compiles literature review from consumer neuroscience field applied to neuromarketing to generate trust within digital social media content. It concludes with three principles to be applied in Neuromarketing in order to generate trust through digital social media; smart purchase feeling, recommendation influence and empathy content which may even generate collective actions.

Keywords: trust, neuromarketing, consumer neuroscience, digital social media

\section{Introducción}

El continuo estudio de aplicación de nuevas tecnologías a las ciencias tradicionales, ha permitido la mejora de nuevas técnicas así como también el surgimiento de nuevos problemas. Para el caso de las ciencias empresariales, el avance científico-tecnológico a partir de las alianzas y aplicaciones del conocimiento (Krafft et al, 2014) desde la neurociencia, y su aplicación en ciencias tradicionales como el marketing, ha permitido la mejora de técnicas de mercadeo, dando paso a la evolución del neuromarketing.

En la alianza del conocimiento entre las ciencias de la informática y las ciencias empresariales, la informática ha permitido la traducción del lenguaje de los negocios tradicionales hacia los negocios electrónicos permitiendo la interacción y mejora en la dinámica con sus usuarios. Sin embargo, con la implementación de la tecnología surgen también nuevas necesidades y 
consideraciones; como la capacidad de absorción del nuevo usuario en el uso de la tecnología (Cohen \& Levinthal, 1990), o la simple diferencia entre las experiencias de usuarios en sitios web de acuerdo a la generación que pertenecen (Djamasbi, 2014).

El desarrollo científico tecnológico ha marcado notoriamente las diferencias generacionales. Cada generación existente; babyboomers, generación X, generación $Y$ (millennials) y la generación $Z$ tienen actitudes y problemas característicos, como resultado de una evolución económica y tecnológica de un continuo desarrollo del conocimiento aplicado a diferentes ámbitos de estudio. Haciendo un énfasis hacia el progreso y sin descuidar la calidad de vida, la innovación y el avance tecnológico de acuerdo a la economía evolucionista son determinantes del crecimiento a largo plazo (Witt, 2008).

Con el desarrollo del conocimiento surgen también problemas propios de la era tecnológica tales como la privatización del conocimiento con la propiedad intelectual, problemas de confianza, memoria y la fragmentación del conocimiento (David \& Foray, 2003).

Por lo que se considera necesario realizar un estudio de los fundamentos del Neuromarketing desde la neurociencia del consumidor para la generación de la confianza en los medios sociales digitales y virtuales, contestando así a la pregunta de investigación ¿Qué aplicaciones de neuromarketing generan confianza en los medios sociales digitales?

\section{NEUROMARKETING}

Definido como la implementación práctica del conocimiento y traído desde la neurociencia del consumidor con propósitos gerenciales (Hubert \& Kenning, 
2008), el neuromarketing, identifica objetivamente el comportamiento del hombre, desde su aspecto cognitivo, de acuerdo al funcionamiento del cerebro y sus neurotransmisores (Lee et al., 2007).

A través del uso de imágenes cerebrales o mediciones tecnológicas que anticipan reacciones del consumidor hacia por ejemplo: el producto, el empaque o la publicidad; los marketistas identifican el entendimiento del comportamiento del consumidor para tomar en cuenta un nuevo error del producto (Schneider \& Woolgar, 2012).

Los dispositivos tecnológicos utilizados en la neurociencia, por ejemplo el eye tracking o el electroencefalograma (EEG) ayudan, a través del neuromarketing, a las compañías a diseñar o mejorar su página web y los medios sociales digitales de acuerdo a la experiencia del usuario, midiendo durante su desarrollo, la aceptación y/o rechazo del mercado objetivo, prediciendo y/o anticipándose a la forma de éxito de campañas publicitarias; estas mediciones ofrecen además ayuda para que después de su lanzamiento, la página web y los medios sociales digitales mantengan su mercado.(Albert \& Tullis, 2013; Djamasbi_et al., 2014).

La neurociencia aplicada al comportamiento económico en la política pública se la conoce como neuroeconomía (Pykett, 2013).

\section{LA NEUROCIENCIA DEL CONSUMIDOR}

El estudio del comportamiento del consumidor desde la neurología se lo conoce como la neurociencia del consumidor (Venkatraman_et al., 2012). La neurociencia del consumidor y el Neuromarketing han contribuido a la neurología en experiencia ganada en investigación clínica y en estándares para 
tratamientos alternativos que no necesariamente refieren al uso exclusivo de medicamentos (Javor et al., 2013). Con los resultados alcanzados de la neurociencia del consumidor, los neurólogos han despertado su interés en estudios de: sistema de recompensa, la confianza y temas éticos que conllevan a tratamientos alternativos de enfermedades mentales. Por ejemplo, el paradigma de la teoría de juegos de la neurociencia del consumidor subraya la patopsicología y los estudios de apostadores patológicos se identificaron como aplicables en la investigación de tratamientos de enfermedades de Parkinson, demencia frontotemporal, epilepsia y la enfermedad de Huntington. Así también, la compra compulsiva ha avanzado el conocimiento de la neurología en estudios de importancia del sistema del comportamiento utilizables en el tratamiento de la enfermedad de Parkinson y la demencia frontotemporal. La investigación en la confianza carecen aún de comportamiento empírico y evidencia neurocientífica, para investigar profundamente sobre sus principios biológicos. (Javor et al., 2013).

El avance de la ciencia ha permitido identificar lo que el consumidor siente, quiere y piensa desde sus orígenes. Sin tomarlo como el establecimiento de un cliente cautivo, se sugiere el enfoque de análisis de la psiconeurobioquímica para el estudio del comportamiento del consumidor ya que permite analizar y sintetizar resultados psicológicos, neurológicos, biológicos, y químicos, como por ejemplo en el caso del marketing turístico, donde se estudian neurotransmisores como la serotonina y dopamina; la hormona melatonina; el fotoperiodo (photoperiod), el ritmo cardíaco y las emociones que son aplicables 
a las 7p's del sector hospitalario para mejorar la fidelidad del cliente (Koc \& Boz, 2014).

El comportamiento del consumidor en la neurociencia clasifica principalmente en cuatro tipos: la toma de decisiones, la recompensa, la memoria y la generación de emociones (Solnais et al., 2013), caracterizándose como principal conductor a sus neurotransmisores, tales como; la serotonina, la dopamina y la oxitocina.

Serotonina:

Las funciones cognitivas y de comportamiento como dormir, estado de ánimo, dolor, adicción, locomoción, actividad sexual, depresión, ansiedad, abuso de alcohol, agresiones y aprendizaje se reflejan en el rol que desempeña el nivel de serotonina en el cerebro. Conocida como la hormona de la felicidad, la serotonina es un neurotransmisor en el cerebro límbico que desempeña funciones de emoción, comportamiento, memoria a largo plazo y olfato, determinada por la disponibilidad del aminoácido tryptophan en el cerebro (Koc $\&$ Boz, 2014).

Dopamina:

La tesis doctoral de Clithero (2011) fue basada en el estudio de la segregación de dopamina y utiliza el fMRI en la aplicabilidad de la neurociencia cognitiva y la economía para estudiar el proceso de información de recompensa y la motivación en el cerebro. A una recompensa inesperada, la dopamina se eleva, a una recompensa esperada la segregación de dopamina en el cerebro no se afecta, y en el caso de una recompensa esperada omitida, la dopamina 
decrece. Es decir, lo que acontece en la zona del striatum del cerebro, es la estructura clave para la neurobiología de la recompensa.

Oxitocina:

La teoría de juegos de Nash, basada en el dilema de los prisioneros, esquematiza la economía principalmente en la no cooperación ni confianza, en donde en un juego repetido infinitamente, los teoremas de tradición oral (folks theorem) sólo funcionan cuando las ganancias a largo plazo exceden las motivaciones individuales de ganancia de corto plazo para lo que debe existir confianza y cooperación en los individuos, que en su dilema no existe.

La confianza puede estar asociada tradicionalmente con la historia psicológica del individuo como con el comportamiento, el estado de ánimo, la cultura, entre otras. Sin embargo, la confianza puede ser considerada también meramente como una reacción química. En un test de modelo neurológico de confianza, poniendo bajo control circunstancias del comportamiento, la confianza es relacionada con los niveles de oxitocina (OT). La liberación de oxitocina activa el circuito del cerebro que, por sus siglas en inglés HOME (Human Oxytocin Mediate Emphaty), causa empatía. Un estudio de esta última afirmación dió como resultado que la liberación de OT permitiera a los participantes enviar dinero a extraños. Evidentemente va en contra de la teoría de Nash que una persona "racional" no enviaría dinero a un extraño (Zak \& Kugler, 2011). Así también la empatía se vuelve un compás interno muy importante para la generación de acciones colectivas desde la investigación de la neuroeconomía y el comportamiento social (Zak \& Barraza, 2013). En otro estudio se identificó también que para generar confianza se debe tomar en cuenta quién 
recomienda la compra, la acción se ejecuta como una respuesta neural de los consumidores durante la compra (Guo et al., 2016).

El especialista en Neuromarketing y la oxitocina Paul J. Zack realiza estudios experimentales para obtener evidencias preliminares que los principios biológicos de la confianza son determinados por la liberación de la oxitocina. La oxitocina es la hormona que la madre libera a su hijo en el periodo de lactancia, se la relaciona con la hormona de compartir, dar amor y así también generar confianza. La hormona es liberada también en el acto sexual. La hormona se la relaciona con el contacto físico solamente, muchos neurólogos coinciden en afirmar "el contacto táctil no puede ser reemplazado en ningún caso por mensajes de texto". Así también, por ejemplo, se relacionan a los masajes con el incremento de la oxitocina y la reducción de la adrenocorticotropina (hormona del estrés) en humanos (Morhenn et al., 2012).

Con lo mencionado anteriormente, se genera entonces la duda de la liberación de oxitocina a través de medios virtuales o redes sociales, por lo que el neurólogo Paul Zack identificó que en un estudio de compras en línea, el ganarse un cupón sorpresa indujo a la liberación de oxitocina sugiriendo esta forma como una alternativa efectiva de crear "amor a la marca". El ahorrar dinero y recibir una buena sensación de una compra inteligente incrementó en un $14 \%$ el nivel de oxitocina en los participantes que fueron controlados al inicio y al final del experimento. El test además produjo la reducción de la adrecorticotropina (hormona del estrés) y variaciones en ratios de corazón, respiración, niveles de conductividad de la piel, entre otros. (Alexander et al., 
2015). La OT induce la liberación de dopamina del cerebro central, generando una sensación de placer (Bale et al., 2001).

\section{Conclusiones y Limitaciones}

De acuerdo a las aplicaciones científicas y tecnológicas de la neurociencia del consumidor aplicadas en neuromarketing, la confianza es generada gracias a la segregación de oxitocina en el cerebro, que de acuerdo a sus autores se genera por; sensaciones de compra inteligente, la influencia de quien realiza la recomendación y los contenidos de empatía que incluso puedan generar acciones colectivas. La revisión bibliográfica no menciona el tiempo ni el número de repeticiones que pueda tomar la generación de confianza a través de estas claves y a la vez sean eficientes con los canales de comunicación, tales como, los medios sociales digitales, ni los efectos que estos causen como para por ejemplo, generar confianza en una publicidad que considere elementos de neuromarketing. La revisión no incluye investigaciones de neurociencia de consumidor aplicada a otros sentidos como el gusto, el olfato y el tacto en la generación de confianza.

\section{Agradecimientos}

Agradecimiento a la Universidad Técnica de Machala por el incentivo a la investigación docente. Un reconocimiento y agradecimiento especial a la mentoría del Dr. Valentín Alejandro Martínez-Fernández, docente titular de la Universidade da Coruña e Investigador del Programa PROMETEO de la 
Secretaría Nacional de Educación Superior, Ciencia y Tecnología (SENESCYT) del Gobierno de Ecuador.

\section{Referencias}

Albert, W., \& Tullis. (2013). Measuring the user experience: Collecting, analyzing, and presenting usability metrics. (M. A. Waltham, Ed.). Morgan Kaufmann.

Alexander, V., Tripp, S., \& Zak, P. J. (2015). Preliminary Evidence for the Neurophysiologic Effects of Online Coupons: Changes in Oxytocin, Stress, and Mood. Psychology and Marketing, 32(9), 977-986.

Bale, T. L., Davis, A. M., Auger, A. P., Dorsa, D. M., \& McCarthy, M. M. (2001). CNS region-specific oxytocin receptor expression: importance in regulation of anxiety and sex behavior. Journal of Neuroscience, 21(7), 2546-2552.

Clithero, J. A. (2011). Neuroeconomics of Reward Information and Motivation. Duke University, Ann Arbor, United States. Retrieved from http://search.proquest.com/pqdtglobal/docview/874146592/abstract/22C89C79006 $34946 \mathrm{PQ} / 26$ ?accountid=17197

Cohen, W. M., \& Levinthal, D. A. (1990). Absorptive Capacity: A New Perspective on Learning and Innovation. Administrative Science Quarterly, 35(1), 128-152.

David, P. A., \& Foray, D. (2003). Economic Fundamentals of the Knowledge Society. Policy Futures in Education. http://doi.org/10.2304/pfie.2003.1.1.7

Djamasbi, S. (2014). Eye Tracking and Web Experience. AIS Transactions on HumanComputer Interaction, 6(2), 16-31.

Djamasbi, S., McAuliffe, D., Gomez, W., Kardzhaliyski, G., Liu, W., \& Oglesby, F. (2014). Designing for success: Creating business value with mobile user experience (UX). Lecture Notes in Computer Science, 8527, 299-306. 
Guo, F., Zhang, X., Ding, Y., Wang, X. (2016). Recommendation influence: Differential neural responses of consumers during shopping online. Journal of Neuroscience, Psychology, and Economics, 9(1), 29-37.

Hubert, M., \& Kenning, P. (2008). A current overview of consumer neuroscience. Journal of Consumer Behaviour, 7(4-5), 272-292.

Javor, A., Koller, M., Lee, N., Chamberlain, L., \& Ransmayr, G. (2013). Neuromarketing and consumer neuroscience: contributions to neurology. BMC Neurology, 13, 13.

Koc, E., \& Boz, H. (2014). Psychoneurobiochemistry of tourism marketing. Tourism Management, 2014(44), 140-148.

Krafft, J., Quatraro, F., \& Saviotti, P. P. (2014). Knowledge characteristics and the dynamics of technological alliances in pharmaceuticals: Empirical evidence from Europe, US and Japan. Journal of Evolutionary Economics. http://doi.org/10.1007/s00191-014-0338-8

Lee, N., Broderick, A. J., \& Chamberlain, L. (2007). What is "neuromarketing"? A discussion and agenda for future research. International Journal of Psychophysiology: Official Journal of the International Organization of Psychophysiology, 63(2), 199-204.

Morhenn, V., Beavin, L. E., \& Zak, P. J. (2012). Massage increases oxytocin and reduces adrenocorticotropin hormone in humans. Alternative Therapies in Health and Medicine, 18(6), 11-18.

Pykett, J. (2013). Neurocapitalism and the new neuros: using neuroeconomics, behavioural economics and picoeconomics for public policy. Journal of Economic Geography, 13(5), 845-869.

Schneider, T., \& Woolgar, S. (2012). Technologies of ironic revelation: enacting consumers in neuromarkets. CONSUMPTION MARKETS \& CULTURE, 15(2), 169-189. 
Solnais, C., Andreu-Perez, J., Sánchez-Fernández, J., \& Andréu-Abela, J. (2013). The contribution of neuroscience to consumer research: A conceptual framework and empirical review. Journal Of Economic Psychology, (36), 68-81.

Venkatraman, V., Clithero, J. A., Fitzsimons, G. J., \& Huettel, S. A. (2012). New scanner data for brand marketers: How neuroscience can help better understand differences in brand preferences. Journal of Consumer Psychology: The Official Journal of the Society for Consumer Psychology, 2012(22), 143-153.

Witt, U. (2008). What is specific about evolutionary economics? Journal Of Evolutionary Economics, 18(5), 547-575.

Zak, P. J., \& Barraza, J. A. (2013). The neurobiology of collective action. Frontiers in Neuroscience, 7, 211.

Zak, P. J., \& Kugler, J. (2011). Neuroeconomics and International Studies: A New Understanding of Trust. International Studies Perspectives, 12(2), 136-152. 